\title{
O professor e o estudante na facoemulsificação: os dez princípios para o sucesso*
}

\author{
The teacher and the student of phacoemulsification: ten principles for success
}

\section{Vinicius Coral Ghanem ${ }^{1}$ Mark J. Mannis ${ }^{2}$}

${ }^{1}$ Fellow do Departamento de Oftalmologia da Universidade da Califórnia, Davis - EUA

${ }^{2}$ Professor do Setor de Córnea e Doenças Externas e Vice-Chefe do Departamento de Oftalmologia da Universidade da Califórnia, Davis - EUA.

* Fontes de auxílio à pesquisa: Medline, Lilacs

Endereço para Correspondência: Rua Fernando de Noronha, 225 apto. 901- Joinville (SC) CEP 89203-070. E-mail: vcghanem@hotmail.com

Recebido para publicação em 08.08.2002 Aceito para publicação em 20.11.2002

\begin{tabular}{|c|}
\hline RESUMO \\
\hline $\begin{array}{l}\text { Neste texto, os autores apresentam os diferentes aspectos do aprendizado } \\
\text { da cirurgia de facoemulsificação pelo cirurgião iniciante. Discutem também } \\
\text { o papel do professor e do aluno no ambiente da "Sala de Aula Cirúrgica", } \\
\text { sugerindo alguns princípios que melhor fundamentam o aprendizado } \\
\text { cirúrgico, reduzindo complicações e proporcionando maior segurança } \\
\text { para o paciente. }\end{array}$ \\
\hline
\end{tabular}

\section{INTRODUÇ̃̃̃O}

"In surgery, as in anything else, skill and confidence are learned through experience - haltingly and humiliatingly. Like the tennis player and the oboist and the guy who fixes hard drives, we need practice to get good at what we do. There is one difference in medicine, though: it is people we practice upon."

[From Complications: A Surgeon's Notes on an Imperfect Science by Atul Gawande, Metropolitan Books, New York, 2002]

O ensinamento cirúrgico representa um desafio, tanto para o orientador, que leva à sala cirúrgica sua experiência e o conhecimento de como lidar com as situações inesperadas, quanto para o cirurgião iniciante, que está meramente tentando aprender os passos básicos de um procedimento. $\mathrm{O}$ cirurgião iniciante deve não apenas compreender os problemas intelectuais e cognitivos que fundamentam um procedimento cirúrgico, mas, também, dominar os aspectos técnicos, com treinamento freqüente no ambiente cirúrgico. Diferente de outras funções cognitivas da medicina, a obtenção bem sucedida da habilidade cirúrgica requer experiência prática, preferencialmente sob supervisão de um cirurgião experiente.

Por outro lado, o paciente que vai a uma sala de cirurgia quer estar confiante nas mãos de um cirurgião, na certeza de que o médico saberá realizar a cirurgia proposta e, conseqüentemente, não lhe causará dano algum. Isso representa um dilema para o estudante e para o professor. Este deve ter muito equilíbrio na hora de permitir ao cirurgião iniciante familiarizar-se com os passos cirúrgicos (habilidade de julgamento e experiência técnica) e, ao mesmo tempo, proteger o paciente dos erros da inexperiência. Essa decisão se torna ainda mais importante no contexto da facoemulsificação, procedimento seqüencial interdependente em que há pouco lugar para erro.

Neste texto, temos como objetivo prover, a estudantes e docentes de facoemulsificação, diretrizes que poderão facilitar o aprendizado do novo cirurgião e proporcionar maior segurança para o paciente. 


\section{UMA BREVE REVISÃO DA LITERATURA}

Nenhuma técnica cirúrgica está isenta de complicações. Espera-se que sejam mais freqüentes quando a cirurgia é conduzida por um cirurgião inexperiente. Entretanto, os resultados apresentados a seguir sugerem que mesmo o cirurgião em treinamento pode obter experiência sem submeter o paciente a um índice de complicações indesejáveis. Em 1992, Cruz et al. ${ }^{(1)} \mathrm{e}$ Allinson et al. ${ }^{(2)}$ relataram os primeiros resultados das cirurgias de facoemulsificação com implante da lente intra-ocular (LIO) realizadas por residentes. No estudo de Cruz et al.(1), em 181 cirurgias, houve ruptura da cápsula posterior (RCP) em $10 \%$ dos casos e perda vítrea (PV) em 5,5\%; enquanto no estudo de Allinson et al. (2) $^{(2)}$ a incidência de PV foi de 14,7\% (20 PV em 136 cirurgias) e a de RCP não foi relatada. Nos dois estudos, as cirurgias foram realizadas por residentes do terceiro ano, com treinamento prévio na técnica de extração extracapsular da catarata (EECC) e sob a supervisão de um cirurgião experiente. Essa diferença de quase três vezes na incidência de PV, entre os estudos, pode ser parcialmente explicada pela seleção pré-operatória inadequada dos pacientes no estudo de Allinson et al. ${ }^{(2)}$. Um terço dos 20 casos de PV ocorreu em situações em que se poderia prever a dificuldade técnica da cirurgia. Não deveriam, portanto, ter sido operados por residentes no início da curva de aprendizado. Cinco desses casos ocorreram em olhos com pupila pequena e dois em olhos com esclerose nuclear de 4+. Após esses estudos, vários outros relataram bons resultados da cirurgia de facoemulsificação realizada por residentes ${ }^{(3-9)}$ (Tabela1), comparáveis aos de cirurgiões experientes, também no início das suas curvas de aprendizado da facoemulsificação ${ }^{(10-13)}$.

Pingree et al. ${ }^{(14)}$ não encontraram diferença significativa na incidência de complicações per-operatórias entre os cirurgiões assistentes, fellows ou residentes, em 1729 cirurgias de facoemulsificação realizadas durante um ano. A incidência da RCPe
PV foi de 2,5\% e 1,7\%, respectivamente. Ionides et al. ${ }^{(15)}$ relataram $1,2 \%$ de RCP em cirurgias realizadas por cirurgiões experientes e 5,3\% em cirurgias realizadas por cirurgiões em treinamento. Neste estudo não está descrito como foi realizada a seleção de pacientes no pré-operatório (densidade nuclear, pupila pequena, etc.) ou qual a orientação per-operatória recebida pelos cirurgiões em treinamento. Apesar dessa diferença, o resultado visual final foi semelhante nos dois grupos.

A análise das complicações pós-operatórias em cirurgias de facoemulsificação, realizadas por residentes, mostra que a opacificação da cápsula posterior (de $0 \%$ a $5 \%$ ), o edema cistóide de mácula (de $0 \%$ a 4,6\%) e a uveíte crônica (de $0 \%$ a $3 \%$ ) foram as mais frequientes ${ }^{(1,4-8)}$. A incidência dessas complicações foi semelhante à dos cirurgiões experientes ${ }^{(16-18)}$. Outras complicações também foram observadas em $1 \%$ ou menos dos casos: hérnia de íris pós-operatória, edema corneal persistente, retenção de material nuclear/cortical intra-ocular, endoftalmite, glaucoma e descolamento de retina.

Embora as complicações per- ou pós-operatórias sejam importantes fatores para a determinação objetiva do sucesso cirúrgico, a acuidade visual (AV) pós-operatória também deve ser considerada nesta análise. Em 1994, Powe et al ${ }^{(16)}$ realizaram uma revisão da literatura sobre os resultados visuais em pacientes submetidos à cirurgia de catarata por meio da técnica de facoemulsificiação. Constataram que entre 69\% e 100\% dos olhos operados atingiram a AV de 0,5 ou melhor no final de seu acompanhamento pós-operatório. E, quando os olhos com alguma comorbidade foram excluídos, esse índice ficou acima dos 90\%. Esses dados são semelhantes aos relatados nos principais trabalhos sobre a cirurgia de facoemulsificação realizada por residentes (Tabela 1).

Entretanto, a avaliação do grau de satisfação do paciente com a cirurgia é mais complexa. Esta engloba uma série de outros fatores, de certa forma subjetivos, como: melhora na qualidade de vida, função visual, condições de saúde geral,

\begin{tabular}{|c|c|c|c|c|c|}
\hline & & & & & \\
\hline cstudo & N Uinos & recnica & nur & PV & $A V=20 / 40$ \\
\hline Lambert et al. ${ }^{(3)} 1997$ & 48 & Faco & $6,25 \%$ & $10,4 \%$ & $89,6 \%$ \\
\hline \multirow[t]{3}{*}{ Smith \& Seiff(4) 1997} & 218 & EECC & & & $78 \%$ \\
\hline & & Faco & $10 \%$ & $8,2 \%$ & $88 \%$ \\
\hline & & & & & $95,8 \%$ * \\
\hline Corey, Olson ${ }^{(5)} 1998$ & 396 & Faco & $2 \%$ & $1,8 \%$ & NR \\
\hline \multirow[t]{2}{*}{ Prasad $^{(6)} 1998$} & 102 & Faco & $5,9 \%$ & $2,8 \%$ & $90,2 \%$ \\
\hline & & & & & $100 \%$ * \\
\hline \multirow[t]{2}{*}{ Badoza et al. ${ }^{(7)} 1999$} & 249 & Faco & $6 \%$ & $2,8 \%$ & $91,2 \%$ \\
\hline & & & & & $99,2 \%$ * \\
\hline Bhan et al. ${ }^{(8)} 2000$ & 37 & Faco $^{\dagger}$ & NR & $3 \%$ & NR \\
\hline \multirow[t]{2}{*}{ Blomquist, Rugwani(9) 2002} & 1400 & EECC & NR & $6,3 \%$ & $42,9 \% \neq$ \\
\hline & & Faco & NR & $4,3 \%$ & $81,5 \% \neq$ \\
\hline
\end{tabular}


satisfação em relação às expectativas pré-operatórias e em relação aos cuidados médicos recebidos durante e após a cirurgia ${ }^{(19-20)}$. Um estudo realizado por Nijkamp et al ${ }^{(19)}$, mostrou que, apesar de $88,8 \%$ dos pacientes terem apresentado melhora na AV pós-operatória, somente $62,7 \%$ relataram melhora na sua qualidade de vida. Os autores concluíram que, para aumentar a satisfação do paciente, maior ênfase deve ser dada aos fatores subjetivos, especialmente no que se refere à orientação pré-operatória e à avaliação das expectativas em relação à melhora da função visual pós-operatória.

Portanto, quando se analisam os principais fatores objetivos que determinam o sucesso cirúrgico, observa-se que o aprendizado da cirurgia de facoemulsificação, apesar de tecnicamente mais difícil que a EECC, pode ser realizado com segurança e com grande índice de sucesso.

\section{OS DEZ PRINCÍPIOS PARA O SUCESSO NO APRENDIZADO DA FACOEMULSIFICAÇÃO}

A cirurgia de catarata é o procedimento cirúrgico oftalmológico mais comumente realizado no Brasil e, provavelmente, no mundo. Portanto, um treinamento qualificado do cirurgião de catarata deve ser um dos principais objetivos de qualquer programa de residência em oftalmologia. A análise periódica dos resultados de cirurgias realizadas por residentes contribui para melhorar a assistência ao paciente e possibilita a autoavaliação por parte do residente e do cirurgião orientador, determinando a real eficácia do programa de treinamento cirúrgico proposto.

Todo treinamento cirúrgico deve começar com o aprendizado teórico, utilizando-se de livros, cursos e vídeos sobre o procedimento a ser realizado. A teoria fundamenta a prática e orienta o cirurgião inexperiente durante as situações inesperadas. Assim, antes de iniciar qualquer procedimento cirúrgico, o principiante deve ter em mente os diferentes aspectos a serem considerados em relação às indicações cirúrgicas, seleção do paciente, anestesia a ser utilizada, técnica cirúrgica proposta, possíveis complicações e formas de evitá-las ou resolvê-las ${ }^{(21)}$.

A seguir, serão apresentados alguns princípios que facilitam o aprendizado do cirurgião iniciante, previnem complicações e aumentam a segurança do paciente.

\section{1) Conhecer a anatomia}

O conhecimento da anatomia local é o primeiro passo no aprendizado cirúrgico. Além disso, reconhecer algumas características anatômicas, como por exemplo: fragilidade capsular encontrada nas cataratas polares posteriores, fragilidade zonular e capsular na pseudoesfoliação, anteriorização da inserção zonular nos pacientes com idade avançada etc, pode ser fator determinante no sucesso cirúrgico.

\section{2) Ter treinamento prévio em cirurgia de facoemulsifica- ção experimental}

A delicadeza dos procedimentos cirúrgicos intra-oculares exige que o cirurgião esteja bem treinado antes de realizar a cirurgia. A mentalização dos passos cirúrgicos é um processo que deve ser adquirido com o treinamento prático em cirurgia experimental. A falta desse treinamento aumenta a ansiedade e insegurança do cirurgião iniciante e do orientador, comprometendo o andamento natural do procedimento.

Talvez uma das maiores dificuldades do cirurgião iniciante é ter domínio sobre a coordenação olhos-ouvidos-mãos-pés, necessária para realizar a facoemulsificação. Os olhos devem estar atentos às manobras cirúrgicas, os ouvidos aos sons produzidos pelo aparelho de facoemulsificação, as mãos coordenadas com a caneta de faco e o instrumento auxiliar, os pés com o pedal do microscópio e o pedal do faco. Está claro que esse é um procedimento cirúrgico mais difícil de dominar e que exige treinamento experimental mais prolongado do que a EECC, em que a coordenação olhos-mãos é suficiente na maioria dos casos. $\mathrm{O}$ treinamento experimental pode ser realizado por meio de "wet-labs", disponíveis amplamente nos congressos e nos cursos específicos de facoemulsificação.

Uma alternativa é a criação de pequenos laboratórios de treinamento, acessíveis a qualquer momento, onde o cirurgião pode aprender, com calma, a manusear os equipamentos e instrumentos cirúrgicos. Os programas de treinamento deveriam ter um "wet-lab" disponível aos residentes, em todos os níveis da curva de aprendizado. Aprender a manipular o microscópio (pedal, "zoom", centragem etc), o aparelho de faco, a caneta e as ponteiras exigem tempo, paciência e orientação.

Além disso, vários passos cirúrgicos podem ser aperfeiçoados com o uso da cirurgia experimental, assim como a capsulorrexes, a emulsificação nuclear e a aspiração cortical, etapas onde a RCP é mais freqüente ${ }^{(2,13,22-23)}$. Diferentes técnicas que induzem ou simulam catarata de densidades variáveis já foram descritas. Olhos humanos "post mortem" (24-25) ou olhos de animais, como porcos $^{(26-27)}$, bois ${ }^{(28)}$ e cabras ${ }^{(29-30)}$, são os mais utilizados, pois simulam uma situação cirúrgica semelhante à real.

Ainda no âmbito da cirurgia experimental, as diversas técnicas de facoemulsificação podem ser aperfeiçoadas. A primeira técnica a ser ensinada é, geralmente, a técnica de "dividir

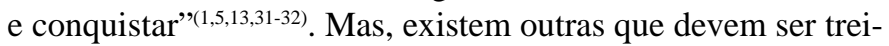
nadas à medida que o cirurgião evolui na curva de aprendizado.

\section{3) Conhecer o equipamento cirúrgico}

A facoemulsificação de sucesso é muito dependente do uso apropriado dos equipamentos cirúrgicos. O conhecimento do aparelho de facoemulsificação facilita a manipulação adequada de suas funções melhorando o resultado.

Com o avanço tecnológico dos aparelhos de facoemulsificação, inúmeros parâmetros foram criados para auxiliar no controle do movimento fluídico intra-ocular e no poder do ultrassom. Situações especiais como núcleos duros, fragilidade zonular e altas ametropias exigem do cirurgião maior controle sobre a cirurgia. Este pode ser conseguido com a programação adequada dos parâmetros, o que aumenta a segurança do procedimento cirúrgico. Portanto, o cirurgião iniciante deve conhecer os parâmetros a serem utilizados e qual a função de cada um deles, não devendo deixar esse aprendizado em segundo plano. 
Centurion ${ }^{(33)}$ sugeriu que o treinamento na técnica de facoemulsificação deve ser dividido em três fases. A fase I tem duração média de duas semanas (seis horas por semana), e envolve o estudo da máquina e suas funções, sem dar importância à técnica cirúrgica. Esta deve ser desenvolvida na fase II, durante o treinamento experimental, com duração média de quatro semanas (seis horas por semana). Portanto, após seis semanas, o cirurgião estaria apto a realizar sua primeira facoemulsificação.

\section{4) Fazer seleção pré-operatória dos pacientes}

Uma seleção pré-operatória cuidadosa é fundamental para reduzir o índice de complicações no início da curva de aprendizado $^{(2,11,13,34)}$. O orientador deve fazer parte da seleção préoperatória dos primeiros pacientes, pois o que é simples para o cirurgião experiente, parece complexo nas mãos do residente, que não consegue prever as dificuldades do procedimento, por falta de experiência prática.

O paciente ideal para se iniciar a curva de aprendizado da facoemulsificação é aquele que se apresenta tranqüilo, cooperativo, com boa exposição ocular, sem patologias de córnea, dilatação pupilar de pelo menos $6 \mathrm{~mm}$, esclerose nuclear moderada e comprimento axial normal.

Em 1996, Gonglore e Smith ${ }^{(35)}$, baseados numa pesquisa envolvendo 14 cirurgiões de facoemulsificação, em diferentes fases da curva de aprendizado, relataram que as principais contra-indicações à realização do procedimento eram: esclerose nuclear avançada, catarata madura, pupila pequena, fragilidade zonular e pacientes com visão monocular. Entretanto, um ano após, os casos de esclerose nuclear avançada e os pacientes com visão monocular eram contra-indicados apenas ocasionalmente. O estudo de Martin e Burton ${ }^{(13)}$, das 3000 primeiras cirurgias de facoemulsificação de um único cirurgião, relatou que a seleção pré-operatória foi realizada somente nos primeiros 200 casos. A partir daí, todos os pacientes foram submetidos à facoemulsificação. Mesmo assim, o índice de RCP caiu de 4,3\% nos primeiros 300 casos, para $0,7 \%$ nos últimos 300. Isso mostra que, apesar da seleção pré-operatória ser fundamental no início da curva de aprendizado, as contraindicações diminuem com o ganho de experiência do cirurgião.

\section{5)Entender a curva de aprendizado da facoemulsificação}

Essa curva é mais prolongada que a da cirurgia de extração extracapsular, por isso, maior número de cirurgias devem ser realizadas em casos considerados "simples" antes de enfrentar os desafiadores.

Cataratas associadas com órbitas profundas, patologias corneais, pequena dilatação pupilar, esclerose nuclear avançada, pseudo-esfoliação, trauma, alta miopia ou hipermetropia, cristalinos subluxados e olhos vitrectomizados devem ser evitados até se obter confiança e reprodutibilidade do procedimento cirúrgico. Essas são situações de risco para RCP, devendo ser reservadas aos cirurgiões experientes ${ }^{(2,31,34-36)}$.

Quantas cirurgias são necessárias até que o cirurgião em treinamento obtenha confiança e reprodutibilidade na facoemulsificação? Não há um número mágico, são fatores que determinam a velocidade do aprendizado. Não só a habilidade individual e a qualidade da orientação recebida no início da curva de aprendizado, mas também o tempo dispendido com o estudo teórico e treinamento experimental. A Tabela 2 mostra os resultados dos principais estudos que comparam as incidências de RCP e PV em diferentes estágios da curva de aprendizado. Observa-se que há uma grande variação no número de cirurgias realizadas até se alcançar a incidência de RCP próxima a $1 \%$. No estudo de Corey e Olson ${ }^{(5)}$ foram necessárias somente 100 cirurgias para reduzir a incidência de RCP de 3,6\% para $0,5 \%$. Mas, no estudo de Martin e Burton ${ }^{(13)}$, a incidência de RCP só ficou próxima a $1 \%$ após terem sido realizadas 1500 cirurgias (considerando-se os resultados obtidos entre as cirurgias de número 600 e 1500) (Tabela 2). Isso pode ter ocorrido porque, após a realização das primeiras 200 cirurgias, não houve mais seleção pré-operatória dos pacientes. Isto é, a partir da cirurgia 201, a técnica de facoemulsificação foi realizada em todos os casos.

\section{6) Optar pela forma de anestesia mais segura}

A anestesia local, peri ou retro-bulbar, ainda é a forma de anestesia mais utilizada pelo cirurgião de facoemulsificação em treinamento, especialmente pelo residente. A anestesia local, quando comparada com a tópica, promove ao cirurgião iniciante maior controle sobre a cirurgia e facilita o manejo das complicações per-operatórias porque permite o uso da incisão limbar ou escleral, provoca acinesia ocular e apresenta maior tempo de efeito anestésico.

A incisão limbar ou escleral facilita a conversão para a técnica de EECC, no caso de RCP associada à presença de

\begin{tabular}{|c|c|c|c|c|c|c|c|c|c|}
\hline Estudo & $\mathrm{N}^{\circ} \mathrm{Cx}$ & 50 & 100 & 200 & 300 & 400 & 600 & 1500 & 3000 \\
\hline Corey, Olson ${ }^{(5)}$ & $\begin{array}{l}\text { RCP } \\
\text { PV }\end{array}$ & $\begin{array}{l}3,6 \% \\
2,6 \%\end{array}$ & $\begin{array}{l}0,5 \% \\
0 \%\end{array}$ & & & & & & \\
\hline Irvine et al. . $^{(12)}$ & RCP & \multicolumn{2}{|c|}{$11 \%$} & \multicolumn{2}{|c|}{$1,5 \%$} & & & & \\
\hline Seward et al. ${ }^{(11)}$ & $\begin{array}{l}\text { RCP } \\
\text { PV }\end{array}$ & & $\begin{array}{l}7,5 \% \\
2 \%\end{array}$ & & & & & & \\
\hline Martin, Burton(13) & $\begin{array}{l}\text { RCP } \\
\text { PV }\end{array}$ & & & & & & & $\begin{array}{l}1,2 \% \\
0,6 \%\end{array}$ & $\begin{array}{l}0,8 \% \\
0,7 \%\end{array}$ \\
\hline
\end{tabular}


grande fragmento nuclear intra-ocular e também é mais apropriada para a implantação das LIO rígidas, geralmente as únicas disponíveis na maioria dos serviços de residência do país.

Muitos cirurgiões que utilizam a anestesia tópica, de rotina, afirmam que a cinesia ocular e palpebral facilita o procedimento cirúrgico, pois permite ao cirurgião orientar o paciente quanto ao posicionamento ocular per-operatório. Entretanto, movimentos oculares súbitos podem ocorrer, aumentando a incidência de complicações, especialmente se o paciente não for cooperativo, ou se a cirurgia estiver sendo conduzida por um cirurgião inexperiente. Mesmo cirurgiões experientes ${ }^{(37)}$ relataram dificuldade per-operatória na realização da facoemulsificação em $6 \%$ dos casos operados sob anestesia tópica, devido à movimentação ocular excessiva. Entretanto, não houve complicações secundárias a esses movimentos.

E por último, o efeito anestésico prolongado permite ao cirurgião realizar a cirurgia com mais tranqüilidade, o que é fundamental no início do aprendizado.

Apesar dessas vantagens da anestesia local, Bhan et al. ${ }^{(8)}$ mostraram que é possível utilizar anestesia tópica, com segurança, mesmo no início da curva de aprendizado da facoemulsificação (Tabela 1). Esse estudo relatou índices de complicações semelhantes em cirurgias realizadas por residentes e por cirurgiões experientes. Deve-se considerar que, o número de cirurgias foi pequeno e nos casos supervisionados o cirurgião orientador realizou todos os passos até a hidrodissecção. Mesmo assim, o grupo operado pelos residentes apresentou um tempo de cirurgia estatisticamente maior (17,9 minutos pelos residentes e 11,8 min pelos orientadores).

Isso levanta algumas questões. Se a cinesia ocular é favorável, por que a incisão, a capsulorrexes e a hidrodissecção foram feitas pelos orientadores? Será que os residentes seriam capazes de realizar o procedimento completo em tempo aceitável para a utilização da anestesia tópica? Considerando o treinamento de residentes, estas são questões que ainda precisam ser respondidas. Conforme, Badoza et $\mathrm{al}^{(7)}$ a facoemulsificação sob anestesia tópica pode ser realizada com sucesso por residentes, após treinamento adequado nas técnicas de EECC, facoemulsificação sob anestesia peribulbar e facoemulsificação sob anestesia sub-tenoniana.

Como a anestesia tópica, provavelmente, será a forma de anestesia mais utilizada na facoemulsificação, o cirurgião iniciante, depois de já estar apto para operar com anestesia local deve, também, fazer seu treinamento nesta técnica.

\section{7) Ter conhecimento da técnica de EECC}

Há autores que acreditam ser necessário o treinamento prévio na cirurgia de $\operatorname{EECC}^{(23,36)}$, antes de se iniciar com a facoemulsificação. O conhecimento teórico e prático básico da cirurgia de extração extracapsular faz com que o cirurgião se sinta mais confortável quando for necessária a conversão da facoemulsificação para a técnica de EECC. Entretanto, esta é uma opinião controversa. Prasad e Kamath ${ }^{(38)}$ afirmam que o aprendizado prévio da técnica de EECC não é mais necessário, e que os residentes orientados por eles iniciam o treinamento cirúrgico com a técnica de facoemulsificação. Mas ressaltam a necessidade de estudos para avaliar a segurança de tal filosofia de treinamento.

Apesar do abandono progressivo da técnica de EECC, os autores que a defendem baseiam-se nos seguintes aspectos: 1) alguns casos, como por exemplo: núcleos extremamente duros, córnea "guttata" avançada e subluxação do cristalino, não são adequados para a facoemulsificação pelo cirurgião iniciante; 2) o aparelho de facoemulsificação pode falhar ou não estar disponível; 3) o residente pode precisar converter para EECC algum caso complicado de facoemulsificação.

Os autores que defendem o ensinamento da facoemulsificação, sem o treinamento prévio na técnica de EECC, baseiamse nos seguintes aspectos: 1) os cirurgiões em treinamento não necessariamente precisam operar os casos complicados, sendo esses reservados aos cirurgiões experientes; 2 ) a técnica de conversão pode ser aprendida quando houver necessidade de utilizá-la, sem ser necessário o treinamento na técnica de EECC, uma vez que a incidência de conversão pelo cirurgião em treinamento é pequena, geralmente, menor que $3 \%(2,7,14) ; 3)$ a incidência de complicações no início da curva de aprendizado da EECC é semelhante ou maior que na técnica de facoemulsificação $0^{(5,7,14,39-42)}$. Portanto, este assunto ainda é controverso e somente novos estudos poderão determinar a segurança de se iniciar a curva de aprendizado da facoemulsificação sem experiência prévia com a EECC.

\section{8) Conhecer as possíveis complicações}

O conhecimento teórico das possíveis complicações per e pós-operatórias auxilia o cirurgião a conduzir os casos inesperados. Como ressaltado anteriormente, a teoria fundamenta a prática.

Com a contínua melhora na qualidade de assistência à saúde e crescente exigência do paciente por bons resultados, as complicações são cada vez menos toleradas. Portanto, recomenda-se estudar o inimigo antes de enfrentá-lo.

\section{9) Ter orientação de um cirurgião experiente}

Esta exigência é fundamental para que as situações de alto risco, na iminência de uma complicação, possam ser contornadas.

É importante a presença do orientador na sala de cirurgia em todos os momentos. A presença física do orientador na sala cirúrgica pode ser um auxílio psicológico ao cirurgião iniciante, mas não é o suficiente para garantir o sucesso do procedimento. $\mathrm{O}$ acompanhamento da cirurgia, de preferência pelo carona do microscópio, é a melhor forma de ensinar e de prevenir complicações. O orientador deve estar com sua atenção voltada à cirurgia, pois muitas vezes o cirurgião iniciante não percebe o perigo iminente que pode levar a uma cascata de complicações.

Além disso, ambos devem dedicar toda atenção ao procedimento que está sendo realizado, evitando as conversas não relacionadas ao procedimento cirúrgico. $\mathrm{O}$ cirurgião em treina- 
mento necessita de toda sua atenção voltada à manobra cirúrgica que está realizando e seus ouvidos devem estar ocupados observando os sons do aparelho de facoemulsificação.

$\mathrm{O}$ orientador deve saber a hora de assumir a cirurgia e o cirurgião deve saber a hora de pedir ajuda. Essa é a melhor forma de ensinar e aprender facoemulsificação.

\section{0) Ser autocrítico}

Gravar e, na companhia do orientador, estudar os casos nos quais ocorreram complicações, além de ser uma excelente forma de aprendizado, diminuirá as chances do cirurgião persistir nos mesmos erros.

Outro importante fator a ser lembrado é que complicações podem acontecer mesmo após cirurgias consideradas tecnicamente perfeitas. Portanto, o paciente deve ser orientado quanto aos cuidados a serem tomados no pós-operatório, medicações a serem usadas, retornos a serem seguidos e sintomas que possam significar complicações (piora da acuidade visual, dor ocular etc.) e que necessitam de avaliação oftalmológica urgente.

Cada passo da cirurgia de catarata, especialmente na facoemulsificação, depende do passo anterior. Conseqüentemente, se os passos prévios não foram bem realizados, os seguintes apresentarão maior dificuldade, com maior chance de complicações. Por exemplo: uma incisão bem construída possibilitará uma câmara anterior bem formada que, associada ao uso de um bom viscoelástico, facilitará a realização de uma capsulorrexes íntegra e de tamanho adequado; conseqüentemente, irá permitir a emulsificação da catarata no saco capsular. Portanto, cada passo deve ser realizado da melhor maneira possível.

Aqui não estão citados todos os princípios da complexa curva de aprendizado da facoemulsificação, entretanto, um não pode ser esquecido: Ter Bom Senso!

Neste texto, foi revisada a literatura relacionada ao cirurgião em treinamento na técnica de facoemulsificação do ponto de vista de um cirurgião experiente e também de um cirurgião em treinamento. Está claro que, na sala cirúrgica, ambos devem enfrentar o desafio.

Os dez princípios propostos aqui podem parecer estar exigindo do estudante de facoemulsificação a apresentação de aprendizado adequado. Entretanto, a responsabilidade de assegurar que habilidades sejam passadas sem risco à segurança do paciente é do professor. Ambos, o experiente e o iniciante, devem focalizar suas atenções no bem-estar do paciente, no contexto da "Sala de Aula Cirúrgica"

\section{ABSTRACT}

In this paper, the authors present different aspects of the phacoemulsification learning process by the new surgeon. They also discuss the professor's and student's role in the "Surgical Classroom", suggesting some principles to improve tutoring of the surgical learning, reducing complications and providing more safety for the patient.
Keywords: Phacoemulsification; Cataract extraction; Postoperative complications; Learning; Clinical competence; Ophthalmology/education; Interprofessional relations; Education based on competence.

\section{REFERÊNCIAS}

1. Cruz OA, Wallace GW, Gay CA, Matoba AY, Koch DD. Visual results and complications of phacoemulsification with intraocular lens implantation performed by ophthalmology residents [commented on Ophthalmology 1992;99:1181]. Ophthalmology 1992;99:448-52.

2. Allinson RW, Metrikin DC, Fante RG. Incidence of vitreous loss among third-year residents performing phacoemulsification [commented on Ophthalmology 1992;99:1181]. Ophthalmology 1992;99:726-30.

3. Lambert LC, Occhiutto ML, Paparelli CM, Kniggendorf S, Akaishi L, Mendonça $\mathrm{BD}$, et al. Resultados visuais e incidência de complicações em facoemulsificação com LIO por residentes. Rev Bras Oftalmol 1997;56:953-6.

4. Smith JH, Seiff SR. Outcomes of cataract surgery by residents at a public county hospital. Am J Ophthalmol 1997;123:448-54.

5. Corey RP, Olson RJ. Surgical outcomes of cataract extractions performed by residents using phacoemulsification [commented on J Cataract Refract Surg 1998;24:727-9]. J Cataract Refract Surg 1998;24:66-72.

6. Prasad S. Phacoemulsification learning curve: experience of two junior trainee ophthalmologists. J Cataract Refract Surg 1998;24:73-7.

7. Badoza DA, Jure T, Zunino LA, Argento CJ. State-of-the-art phacoemulsification performed by residents in Buenos Aires, Argentina [commented on J Cataract Refract Surg 2000;26:794-5]. J Cataract Refract Surg 1999;25:1651-5.

8. Bhan A, Squirrel D, Longstaff S. Teaching junior ophthalmologists phacoemulsification under topical anaesthesia. Eye 2000;14(Pt 5):810-1.

9. Blomquist PH, Rugwani RM. Visual outcomes after vitreous loss during cataract surgery performed by residents. J Cataract Refract Surg 2002;28:847-52.

10. Dantas PEC, Dantas MCN, Mandia Jr C, Waiswol M, Krasilchik G, Dias AKG. Facoemulsificação: a experiência da conversão; análise dos primeiros casos. Arq Bras Oftalmol 1995;58:421-4.

11. Seward HC, Dalton R, Davis A. Phacoemulsification during the learning curve: risk/benefit analysis. Eye 1993;7(Pt 1):164-8.

12. Irvine S, Francis IC, Kappagoda MB, Haylen MJ, Alexander S, Schumacher RS, et al.. The second two hundred cases of endocapsular phacoemulsification: the learning curve levels off. Aust N Z J Ophthalmol 1994;22:281.

13. Martin KR, Burton RL. The phacoemulsification learning curve: per-operative complications in the first 3000 cases of an experienced surgeon. Eye 2000;14(Pt 2):190-5.

14. Pingree MF, Crandall AS, Olson RJ. Cataract surgery complications in 1 year at an academic institution. J Cataract Refract Surg 1999;25:705-8.

15. Ionides A, Minassian D, Tuft S. Visual outcome following posterior capsule rupture during cataract surgery. Br J Ophthalmol 2001;85:222-4.

16. Powe NR, Schein OD, Gieser SC, Tielsch JM, Luthra R, Javitt J, et al. Synthesis of the literature on visual acuity and complications following cataract extraction with intraocular lens implantation. Cataract Patient Outcome Research Team [Commented on Arch Ophthalmol 1994;112:875]. Arch Ophthalmol 1994;112:239-52.

17. Riley AF, Malik TY, Grupcheva CH, Fisk MJ, Craig JP, McGhee CN. The Auckland Cataract Study: co-morbidity, surgical techniques, and clinical outcomes in a public hospital service. Br J Ophthalmol 2002;86:185-90.

18. McKellar MJ, Elder MJ. The early complications of cataract surgery: is routine review of patients 1 week after cataract extraction necessary? Ophthalmology 2001;108:930-5.

19. Nijkamp MD, Nuijts RM, Borne Bvd, Webers CAB, Van der Horst F, Hendrikse F. Determinants of patients satisfaction after cataract surgery in 3 settings. J Cataracst Refract Surg 2000;26:1379-88.

20. Chang-Godinich A, Ou RJ, Koch DD. Functional improvement after phacoemulsification cataract surgery. J Cataract Refract Surg 1999;25:1226-31.

21. Dreyer EB, Volpe NJ. Eleven commandments for teaching ophthalmic surgery. Ophthalmology 2000;107:415-6.

22. Vajpayee RB, Sharma N, Dada T, Gupta V, Kumar A, Dada VK. Management of posterior capsule tears. Surv Ophthalmol 2001;45:473-88.

23. Araújo MEXS, Chou A, Silva CR, Oliveira LB, Neustein I. Facoemulsificação: resultados e complicações nos primeiros 100 olhos. Arq Bras Oftalmol 2000; 63:29-31. 
24. Liu ES, Eng KT, Braga-Mele R. Using medical lubricating jelly in human cadaver eyes to teach ophthalmic surgery. J Cataract Refract Surg 2001; 27:1545-7.

25. Pandey SK, Werner L, Escobar-Gomez M, Visessook N, Peng Q, Apple DJ. Creating cataracts of varying hardness to practice extracapsular cataract extraction and phacoemulsification(2). J Cataract Refract Surg 2000;26:322-9.

26. Mekada A, Nakajima J, Nakamura J, Hirata H, Kishi T, Kani K. Cataract surgery training using pig eyes filled with chestnuts of various hardness. J Cataract Refract Surg. 1999;25:622-5.

27. Sugiura T, Kurosaka D, Uezuki Y, Eguchi S, Obata H, Takahashi T. Creating cataract in a pig eye. J Cataract Refract Surg. 1999;25:615-21.

28. Coroneo MT. The bovine eye as a model for the novice cataract surgeon. Ophthalmic Surg 1990;21:772-7.

29. Dada VK, Sindhu N. Cataract in enucleated goat eyes: training model for phacoemulsification. J Cataract Refract Surg 2000;26:1114-6.

30. Sudan R, Titiyal JS, Sethi HS, Rai HK. Animal model for teaching phacoemulsification [commented on J Cataract Refract Surg 2001;27:814-6]. J Cataract Refract Surg 2002;28:4-5.

31. Snyder RW, Donnenfeld ED. Teaching phacoemulsification to residents and physicians in transition. Int Ophthalmol Clin 1994;34:191-9.

32. Smeets B, Tabandeh H, Teimory M, Seward H. Learning phaco in a teaching environment. Bull Soc Belge Ophtalmol 1993;249:23-8.
33. Centurion V. Facoemulsificação sem traumas ou como se iniciar Faco com o mínimo de stress. Rev Bras Oftalmol 1994;53:83-5.

34. Khouri AS, Uwaydat SH. Patient selection for phacoemulsification by residents in underdeveloped countries. J Cataract Refract Surg 1998;24:148-9.

35. Gonglore B, Smith R. Extracapsular cataract extraction to phacoemulsification: why and how? [commented on Eye 1999;13(Pt 3a):392]. Eye 1998;12(Pt 6):976-82.

36. Cotlier E. Phacoemulsification by residents [commented on Ophthalmology 1992;99:726-30]. Ophthalmology 1992;99:1481-2.

37. O’Brien PD, Fulcher T, Wallace D, Power W. Patient pain during different stages of phacoemulsification using topical anesthesia. J Cataract Refract Surg 2001;27:880-3.

38. Prasad S, Kamath GG. Phacoemulsification performed by residents. J Cataract Refract Surg 2000;26:794-5.

39. Pearson PA, Owen DG, Van Meter WS, Smith TJ. Vitreous loss rates in extracapsular cataract surgery by residents. Ophthalmology 1989;96:1225-7.

40. Straatsma BR, Meyer KT, Bastek JV, Lightfoof DO. Posterior chamber intraocular lens implantation by ophthalmology residents: a prospective study of cataract surgery. Ophthalmology 1983;90:327-35.

41. Browning DJ, Cobo M. Early experience in extracapsular cataract surgery by residents. Ophthalmology 1985;92:1647-53.

42. Ohrloff C, Zubcov AA. Comparison of phacoemulsification and planned extracapsular extraction. Ophthalmologica 1997;211:8-12. 